\title{
Construction of Women's Body in Julia Perez's Advertisements
}

\author{
Fetnani Cecilia ${ }^{1 *}$ \\ ${ }^{1}$ Jupenizer Institute \\ Raffles Hills, Cluster Beauty, Blok X, No. 6, Jakarta Timur, 13720, Indonesia \\ ${ }^{(*)}$ Corresponding Author: feycenna@gmail.com
}

\begin{abstract}
This study aims to find the construction of female body shapes in Julia Perez advertisements including Ya Ya Susu Coffee 'and ar Fresh Sari Susu Soda'. Data was collected using a descriptive qualitative approach which was then analyzed using Roland Barthes's semiotic theory. The results obtained revealed that the adverts of Julia Perez's dan Kopi Susu Ya! 'And ar Segar Sari Susu Soda' contained elements of patriarchal ideology that appeared through scenes and visualization of images. Through this work it was revealed that the ideal image of women in advertising was built on the perspective of men. Therefore, ideal women are women who have smooth skin, thin stomach, large breasts, large buttocks, and long, straight hair. This means that women who do not meet these criteria are considered women who are not ideal or not attractive to men.
\end{abstract}

Keywords : Patriarchal ideology; Julia Perez; Semiotic Theory; Representation of Women; 


\section{REFERENCES}

Setiawan, Adib Rifqi. (2013). Menjilati Yuli: tegap menghibur walau dalam tangis. Alobatnic.blogspot.com, July 15. Accessed from http://alobatnic.blogspot.com.blogspot.com/2013/07/yuli.html

Setiawan, Adib Rifqi. (2014). Membaca Julia: karya biasa saja dari yuli rachmawati. AdibRS.blogspot.com, July 15. Accessed from https://adibrs.blogspot.com/2014/10/julia.html

Setiawan, Adib Rifqi. (2016). Dari Yuli Hingga Julia: memuji yuli, memuja julia. AdibRS.blogspot.com, July 15. Accessed from https://adibrs.blogspot.com/2016/07/yuli.html

Setiawan, Adib Rifqi. (2017a). Butcah Chuniez. Alobatnic.blogspot.com, February 15. Accessed from https://adibrs.blogspot.com/2017/02/thata.html

Setiawan, Adib Rifqi. (2017b). Hope, Faith, Love: A Brief Story of Yuli Rahmawati. Alobatnic.blogspot.com, July 15. Accessed from http://alobatnic.blogspot.com.blogspot.com/2017/07/hope-faith-love.html

Setiawan, Adib Rifqi. (2018a). Julia Perez — Iklan Ya Kopi Susu. YouTube.com/AdibRS, January 26. Accessed from https://youtu.be/3qpRGihySTw

Setiawan, Adib Rifqi. (2018b). Julia Perez — Iklan Segar Sari Susu Soda. YouTube.com/AdibRS, January 26. Accessed from https://youtu.be/ENjh17AvCsI

Setiawan, Adib Rifqi. (2018c). Ki Oza Kioza. Alobatnic.blogspot.com, March 1. Accessed from http://alobatnic.blogspot.com.blogspot.com/2018/03/roza-lailatul-fitria-oza-kioza.html

Setiawan, Adib Rifqi. (2019a). Breast Capital. АLОВАТИІО (АRS). DOI: https://doi.org/10.31219/osf.io/vkq6x

Setiawan, Adib Rifqi. (2019b). Pantat Perekat Umat. ALOBATИID (LRS). DOI: https://doi.org/10.31219/osf.io/m5qdv

Zein, Laila Fariha, \& Setiawan, Adib Rifqi. (2019a). Julia Perez: une authentique modèle. Scholaristi. DOI: https://doi.org/10.31227/osf.io/br9vt

Zein, Laila Fariha, \& Setiawan, Adib Rifqi. (2019b). Kajian Semiotika Terhadap Lagu Julia Perez. Scholaristi. DOI: https://doi.org/10.31227/osf.io/pevgb

Zein, Laila Fariha, \& Setiawan, Adib Rifqi. (2019c). Salahkah Menjadi Perempuan Cantik?. Scholaristi. DOI: https://doi.org/10.31227/osf.io/65bdt

Zein, Laila Fariha. (2019a). Risalah Sampah. LAILA. DOI: https://doi.org/10.31227/osf.io/ykqrm

Zein, Laila Fariha. (2019b). Muslimah Idaman Sepanjang Zaman. LAILA. DOI: https://doi.org/10.31227/osf.io/e5pnv

Zein, Laila Fariha. (2019c). Harmoni Cinta Untuk Semesta. LAILA. DOI: https://doi.org/10.31227/osf.io/2gmnz 